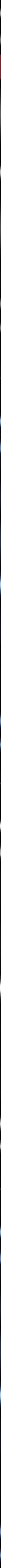


4 P.13: A gallery of the modes guided in a hollow core $\mathrm{PCF}$

\section{Nanometres and femtoseconds}

Left to its own devices, light scatters everywhere. It is the ultimate radar, exploring every cubic micron of a room in a tiny fraction of a second and relaying the information almost instantaneously to the eye. Since an optical cycle is only a few femtoseconds in duration, light can at the same time be used to follow some of the fastest events in the natural world. It can thus be used to probe phenomena in four dimensions - three spatial and one temporal-with astonishingly high resolution. Its ability to follow ultrafast events also means that it can be used to encode information at ultra-high data rates. Understanding, taming and making use of these extraordinary properties has occupied scientists for hundreds of years, culminating in the invention of the laser over 50 years ago, a breakthrough that has stimulated for example the creation of the world-wide-web, whose interconnecting strands are glass optical fibre waveguides capable of transmitting signals at terabit rates under oceans and across continents, and laser machining, which has transformed precision manufacturing world-wide.

\section{Bending light around corners}

The success of optical fibre lies in its ability to overcome the natural tendency of light to diffract and travel in straight lines in free space (although occasionally it takes a curved path, for example when reflected by an inversion layer of hot air trapped on a sun-baked road surface, giving rise to a mirage). In 1841 Swiss scientist Jean-Daniel Colladon, by directing a beam of sunlight into a stream of water issuing from a tank, was the first to show scientifically that total internal reflection could be used to force light to turn a corner [1]. Irishman John Tyndall further popularised this experiment in a demonstration at the Royal Institution in London in 1870. Glass fibre optics is a lineal descendant of these early experiments, and its history makes fascinating reading [2], starting with the pioneering work of Snitzer [3] and followed shortly afterwards by Kao's 1966 prediction that glass fibres could be used for telecommunications [4].

\section{Integrated optical circuitry}

The arrival of the laser, a source of coherent optical radiation not dissimilar in nature to a pure microwave or radio signal but at a much higher frequency, inspired scientists in the late 1960s at Bell Laboratories in the USA to explore trapping light in thin films and strips of dielectric material placed on a low index substrate [5]. By integrating many components on one platform, they aimed to miniaturise bulk optics in much the same way as electronic components have been integrated on to microprocessor chips. This led to the invention of devices such as prism and grating couplers, wavelength division multiplexers, Bragg mirrors and distributed feedback lasers (many of these were inspired by microwave devices).

Integrated optical components are now routinely used in the rapidly growing field of silicon photonics, where the remarkable facilities in silicon foundries are applied to the manufacture of integrated optical chips.

One of the members of the original Bell Labs team was Reinhard Ulrich, who in 1970 moved to the Max Planck Institute for Solid-State Research in Stuttgart, Germany, where in 1975 (stimulated by Brillouin's book on wave propagation in periodic structures [6]) he embarked on a set of beautiful experiments involving the often-counter-intuitive behaviour of visible photonic Bloch waves in thin dielectric films etched with multiply periodic structures (Fig. 1). Borrowing tools from electronic band structure, he and his group used this elegant two-dimensional system to observe effects such as negative \& positive refraction, zero diffraction and the exquisite field patterns created by the interference of guided Bloch waves-topics that are closely related to (and predate) the fields of photonic crystals and metamaterials [7] [8]. The field of guided wave "photonic crystals" is now very active, though the pioneering early work of Ulrich's group is not often recognised.

The biggest success story of guided wave optics is, however and of course, single-mode fibre (SMF) used in optical communications. Perfected in the 1980s, and offering extremely low transmission losses $(\sim 0.2 \mathrm{~dB} /$ $\mathrm{km}), \mathrm{SMF}$ has close to ideal properties. It has also found 

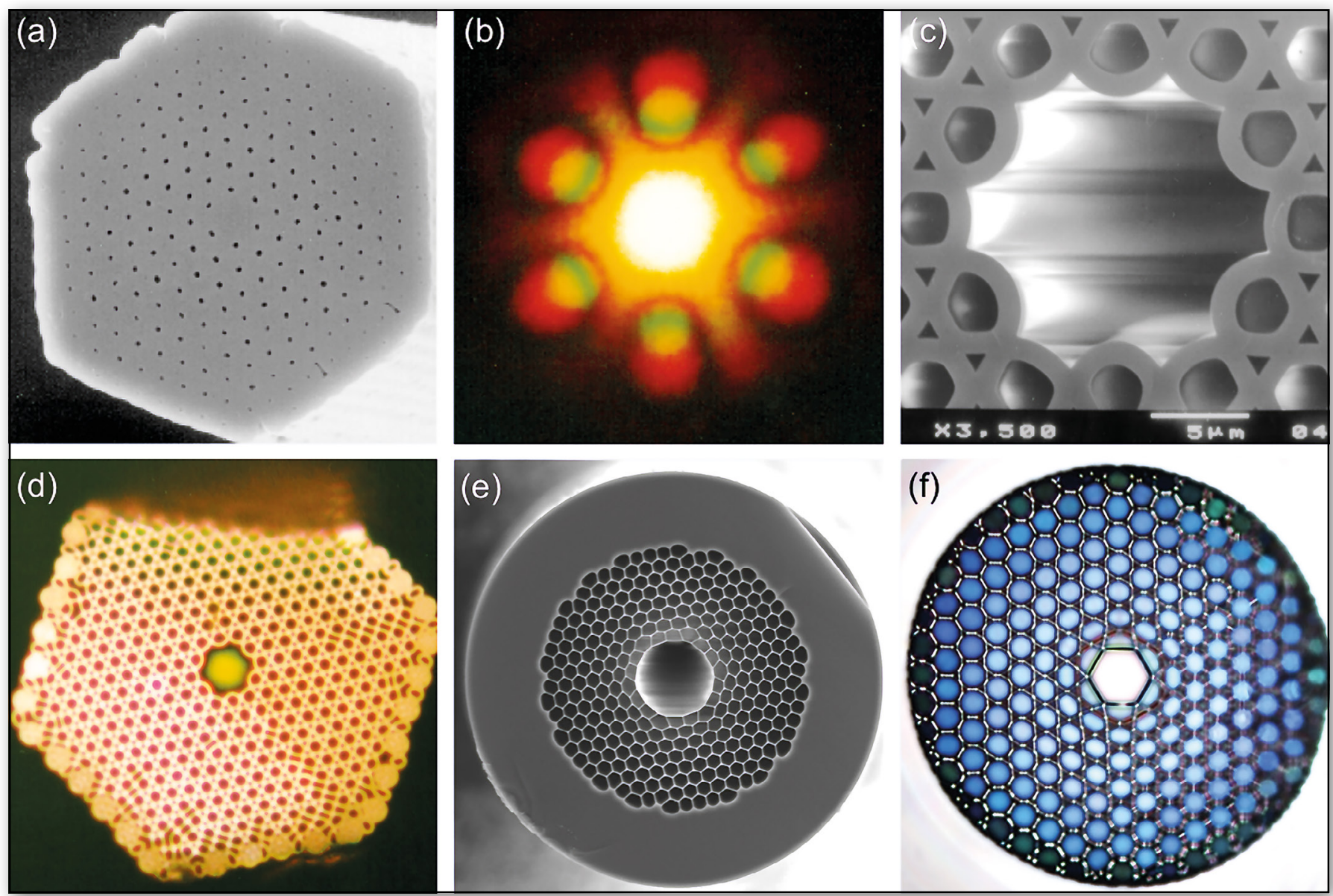

\FIG. 2: Gallery of PCF structures. (a) The first solid-core PCF. (b) Far-field pattern of endlessly- single-mode PCF guiding white light. (c) The first hollow-core PCF and (d) the near-field pattern at its endface when white light was launched. (e) State-of-the-art hollow core PBG PCF. (f) Kagomé-style hollow core PCF guiding white light.

important uses in structural monitoring of, e.g., buildings, ships, aircraft, bridges, chemical plants and oil wells. Such fibre sensors make use of miniature Fabry-Pérot and Mach-Zehnder resonators, Bragg and long-period fiber gratings-and nonlinear optical effects such as Brillouin and Raman scattering. Indeed, experimental nonlinear optics has itself benefitted hugely from the availability of SMF, which made possible for the first time the observation of many effects within a near-perfect one-dimensional system that offers diffraction-free propagation at high intensities while allowing precise tailoring of the dispersion.

\section{Emergence of holey (with an 'e') fibres}

By 1991, fibres seemed as close to perfect as anyone could wish. Nevertheless, the story was not at an end. At that time it was suggested that low-loss guidance of light in a hollow core might be possible if one could create a two-dimensional "photonic bandgap" (PBG) crystal of microscopic hollow channels in the cladding of an optical fiber [9]. The challenge would be to design a suitable structure and, not least, work out a way of making it.

After several attempts in the period 1991 and 1995, the first working photonic crystal fibre (PCF) was drawn from a preform constructed by stacking 216 silica capillaries in a tight-packed hexagonal array around a central solid core (Fig. 2). The fibre guided by a kind of modified total internal reflection and led to the discovery of "endlessly single-mode" PCF which, if it guides at all, only supports the fundamental mode [9].

\section{In endlessly single-mode PCF, the fundamental modes of the glass channels around the core have refractive indices lower than the fundamental core mode, which is therefore trapped by the equivalent of total internal reflection. Higher or- der core modes, on the other hand, have lower indices and are able to leak away between the hollow channels. This modal filtering effect was in fact predicted in an early 1974 paper on sin- gle-material fibres [10].}

\section{Group velocity dispersion}

It was soon realised that, compared to SMF, solid-core PCF offers much greater control over group velocity dispersion (GVD) - the effect that causes the velocity of a pulse to change with its central frequency. 
Consider a hollow planar waveguide formed by two flat mirrors spaced d apart. Rays of light can be trapped by zig-zagging to and fro between them, and there seems no particular reason why certain angles should be favoured. As d gets smaller, however, the wave nature of light begins to reveal itself. Upward and downward travelling rays interfere, producing a pattern of fringes, parallel to the mirrors, with a spacing that depends on the ray angle $\theta$ (see Fig. 3 ). When only one fringe fits between the mirrors, the waveguide is resonant with the light and a fundamental mode forms. If the vacuum wavelength $\lambda$ falls, $\theta$ has to fall to maintain the same fringe width, which reduces the number of bounces per metre and causes the group velocity to increase (at the same time the phase velocity decreases, causing the modal refractive index to rise).

A hollow waveguide has anomalous GVD, i.e., pulses at a higher (bluer) frequencies always travel faster (Fig. 3). If the core is filled, however, its anomalous dispersion is either reinforced or reduced, depending on the GVD of the filling material.

\section{0,000 times brighter than the sun}

In PCFs with micron-sized solid glass cores surrounded by hollow channels, the geometrical dispersion is strongly

v FIG. 3: (a) Fundamental mode of a narrow waveguide is formed by the interference of upward and downward rays. (b) For shorter wavelengths the number of bounces per unit length falls, resulting in an increase in group velocity. (c) Solid-core PCF with very strong anomalous waveguide dispersion, permitting the $1.29 \mu \mathrm{m}$ zero dispersion wavelength of silica to be shifted into the near IR. (d) Iconic photograph of supercontinuum generation in a silica PCF (taken by Will Reeves when he was a PhD student at the University of Bath).
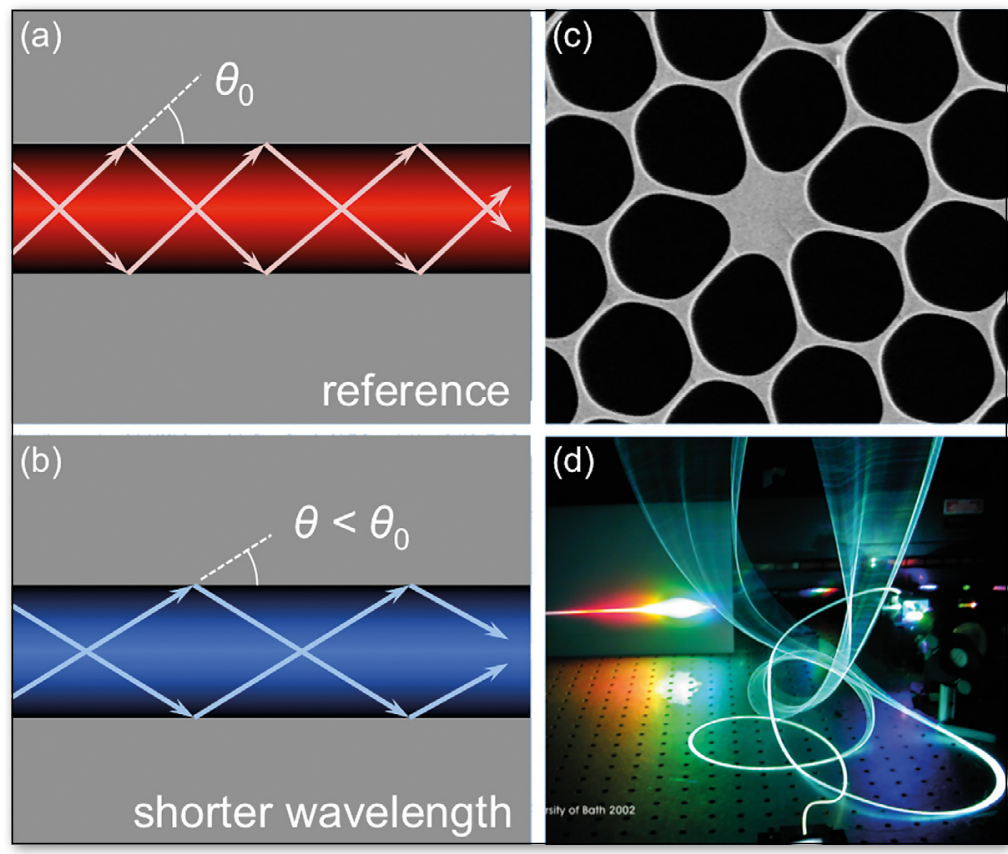

anomalous, which counterbalances the normal dispersion of the glass and shifts the zero dispersion wavelength from $1.29 \mu \mathrm{m}$ (its value for silica-based SMF) into the visible. This creates ideal conditions for generating octave-spanning supercontinua (Fig. 3), and forms the basis for a revolutionary range of extremely bright white-light sources [11], with multiple applications from frequency metrology to hyperspectral imaging [12]. Recently a dispersion-tailored small-core PCF was drawn from the fluorozirconate glass ZBLAN and used to generate a spectrum extending from $200 \mathrm{~nm}$ to beyond $2 \mu \mathrm{m}$ in wavelength (Fig. 4). A remarkable feature of this source is that, unlike silica-glass, the ZBLAN glass shows no sign of degradation in the UV, even when operating for many hours at high brightness [13].

\section{Gas, glass and ultrafast light}

As a result of self-focusing through the electronic Kerr effect (which causes the refractive index to increase with intensity), a laser beam can reach field strengths sufficient to ionise the gas. The release of free electrons creates a negative index change that opposes the Kerr effect. The resulting balance of focusing and defocusing, together with the GVD of the medium, leads to self-guidance of light (known as "filamentation" [14]) and provides a means of channelling a laser beam up into the atmosphere to probe, e.g., for environmental pollutants.

Hollow core PCF offers for the first time a low-loss linear means of guiding light in a narrow $(\sim 20 \mu \mathrm{m})$ channel filled with gas (Fig. 2). This has triggered the emergence of a new generation of versatile and efficient gas-based nonlinear devices, based on "anti-resonant-reflecting" (ARR) PCFs that do not possess a full two-dimensional PBG but provide ultra-broad-band guidance at losses of $1 \mathrm{~dB} / \mathrm{m}$ or less. Examples of ARRPCFs include structures with a kagomé lattice cladding or a single ring of hollow channels around the central hollow core. ARR-PCFs are particularly suited to ultrafast nonlinear optics because they can accommodate the bandwidth needed to support fs pulses, and-a first for fibre optics-the GVD can be adjusted simply by changing the gas pressure. Since more than $99.9 \%$ of the light travels in the hollow core, they have a damage threshold of more than $10^{14} \mathrm{~W} / \mathrm{cm}^{2}$, some 100 times higher than in glass. These features have led to a number of dramatic results, including efficient generation of few-cycle pulses by soliton self-compression of $\sim 1$ $\mu \mathrm{J}, \sim 30$ fs pump pulses, and bright widely-tunable UV light sources extending deep into the vacuum UV [15].

\section{Twisted light}

Solid-core PCFs continue to find new applications. For example, the non-circular transverse microstructure has a remarkable effect when a PCF is twisted 
continuously (by thermal post-processing or by spinning the preform during fibre drawing). This creates orbital angular momentum (OAM) states in the cladding that couple to the core mode, creating sharp dips in the transmitted spectrum at wavelengths that scale with the twist rate - an effect that can be used to measure mechanical twist. Twisted PCFs with cores placed in a ring equidistant from the axis have the unique property of preserving the magnitude and sign of the orbital angular momentum over long distances, i.e., they are OAM-birefringent [16].

\section{Son et lumière}

Another emerging field is that of optoacoustic devices, where the light itself drives mechanical resonances in the glass core. The tight confinement of both acoustic and optical energy within a small PCF core creates a very strong interaction. One of the first experiments in this direction involved pumping the $\mathrm{GHz}$ acoustic core resonance in a PCF with two laser signals, spaced apart in frequency by the acoustic frequency. The action and back-action of sound on light resulted in the generation of a GHz frequency comb. This effect has recently been used to stably mode-lock a fibre soliton laser at the $126^{\text {th }}$ harmonic $(2.1 \mathrm{GHz})$ of its round-trip frequency (16.8 $\mathrm{MHz}$ ) [17]. These ideas are now being explored in the context of silicon integrated optics [18].

\section{Concluding remarks}

In this short account it has been not possible to cover all aspects of optical waveguides-I have focused mainly on photonic crystal fibres. Perhaps, though, it is worth mentioning that hollow core PCF has been proposed as a "low-latency" means of transmitting information, since optical signals travel $\sim 1.5$ times more quickly in air [19]. Applications include high frequency stock-market trading and communications between high performance computers. Overall, it is certain that waveguides, as a vital ingredient in state-of-the-art optics and photonics, will continue to play a key role in fundamental and applied research, as well as commercial products, for the foreseeable future.

\section{About the Author}

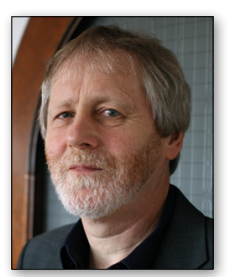

Philip Russell is a founding Director at the Max-Planck Institute for the Science of Light (MPL), a position he has held since January 2009. He obtained his D. Phil. (1979) degree at the University of Oxford. His interests currently focus on scientific applications of photonic crystal fibres. He is a Fellow of the Royal Society and the Optical Society of America (OSA) and has won several awards including the 2000 OSA Joseph Fraunhofer Award/Robert M. Burley Prize, the 2005 Thomas Young Prize of the IOP, the 2005

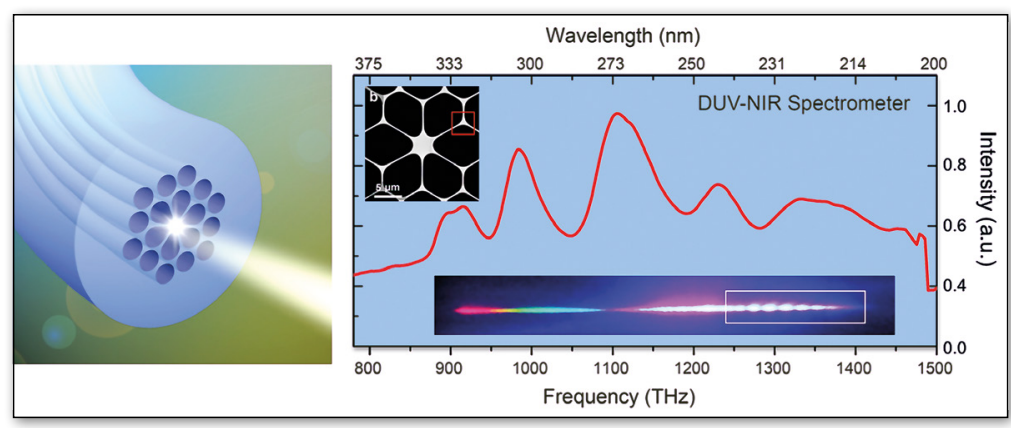

$\triangle$ FIG. 4: A remarkably bright and stable band of deep ultraviolet light is generated in a small-core ZBLAN PCF with carefully tailored dispersion.

Körber Prize for European Science, the 2013 EPS Prize for Research into the Science of Light, the 2014 Berthold Leibinger Zukunftspreis and the 2015 IEEE Photonics Award. He is OSA's President in 2015, the International Year of Light.

\section{References}

[1] D. Colladon, Comptes Rendus 15, 800 (1842).

[2] J. Hecht, City of Light (Oxford University Press, 1999).

[3] H. Osterberg, E. Snitzer, M. Polanyi, R. Hilberg, and J. W. Hicks, J. Opt. Soc. Am. 49, 1128 (1959).

[4] K. C. Kao and G. A. Hockham, Proc. IEE - London 113, 1151 (1966).

5] S. E. Miller, Bell Syst. Tech. J. 48, 2059 (2013).

[6] L. Brillouin, Wave Propagation in Periodic Structures: Electric Filters and Crystal Lattices (McGraw-Hill, 1946).

[7] R. Zengerle, Journal of Modern Optics 34, 1589 (1987).

[8] P. St.J. Russell, Electron and Photon Confinement in Semiconductor Nanostructures (IOS Press, 2003), pp. 79-103.

[9] P. St.J. Russell, Science 299, 358 (2003).

[10] P. V. Kaiser and H. W. Astle, Bell Syst. Tech. J. 53, 1021 (1974).

[11] J. M. Dudley, G. Genty, and S. Coen, Rev. Mod. Phys. 78, 1135 (2006).

[12] C. F. Kaminski, R. S. Watt, A. D. Elder, J. H. Frank, and J. Hult, Appl. Phys. B 92, 367 (2008).

[13] X. Jiang, N. Y. Joly, M. A. Finger, F. Babic, G. K. L. Wong, J. C. Travers, and P. St.J. Russell, Nat. Phot. 9, 133 (2015).

[14] L. Berge, S. Skupin, R. Nuter, J. Kasparian, and J. P. Wolf, Reports on Progress in Physics 70, 1633 (2007).

[15] P. St.J. Russell, P. Hölzer, W. Chang, A. Abdolvand, and J. C. Travers, Nat. Phot. 8, 278 (2014).

[16] X. M. Xi, G. K. L. Wong, M. H. Frosz, F. Babic, G. Ahmed, X. Jiang, T. G. Euser, and P. St.J. Russell, Optica 1, 165 (2014).

[17] M. Pang, X. Jiang, W. He, G. K. L. Wong, G. Onischukov, N. Y. Joly, G. Ahmed, C. R. Menyuk, and P. St.J. Russell, Optica 2, 339 (2014).

[18] R. Van Laer, B. Kuyken, D. Van Thourhout, and R. Baets, Nat. Phot. 9, 19 (2015).

[19] F. Poletti, N. V. Wheeler, M. N. Petrovich, N. Baddela, E. N. Fokoua, J. R. Hayes, D. R. Gray, Z. Li, R. Slavik, and D. J. Richardson, Nat. Phot. 7, 279 (2013). 